\title{
Medicinal plants in the cultural landscape of a Mapuche-Tehuelche community in arid Argentine Patagonia: an eco-sensorial approach
}

Soledad Molares ${ }^{1}$ and Ana Ladio ${ }^{2^{*}}$

\begin{abstract}
Background: The taste and smell of medicinal plants and their relation to the cultural landscape of a Mapuche-Tehuelche community in the Patagonian steppe was investigated. We assume that the landscapes as a source of therapeutic resources is perceived, classified and named according to different symbolic, ecological and utilitarian criteria which are influenced by chemosensorial appearance of medicinal plants which are valued by inhabitants.

Methods: Information relating to the cultural landscape experienced by 18 inhabitants, all representing $85 \%$ of the families, in terms of medicinal plants, knowledge of species and their organoleptic perception was obtained through participant observation, interviews and free listing. The data were examined using cualitative and quantitative approach, including discourse analysis and non-parametric statistics.

Results: Informants use 121 medicinal species, obtained from both wild and non-wild environments, most of which (66\%) present aroma and/or taste. It was found that the plants with highest use consensus used for digestive, respiratory, cardio-vascular, analgesic-anti-inflammatory, obstetric-gynaecological and genito-unrinary complaints, have the highest frequencies of cites reporting flavor; and those with the highest frequencies relating to digestive, analgesic-anti-inflammatory and cultural syndromes present the highest frequencies of aroma. Flavor and/or aroma are interpreted as strong or soft, and the strongest are associated with treatment of supernatural ailments. Also, taste is a distinctive trait for the most of the species collected in all natural units of the landscape, while aroma is more closely associated with species growing at higher altitudes. The local pharmacopeia is also enriched with plants that come from more distant phytogeographical environments, such as the Andean forest and the Patagonian Monte, which are obtained through barter with neighboring populations. Herbal products are also obtained in regional shop. The practices of barter and purchase extend the limits of the landscape as a provider of therapeutic resources, improving the dynamics of its functions and structure, leading to more effective solutions to the various health needs that arise in the community.
\end{abstract}

Conclusions: Herbal landscape perceived by the community exhibits notable eco sensorial and spatial heterogeneity. Local inhabitants' sensorial interpretations play a role as heuristic tools in the recreation and redefinition of old and new available resources.

Keywords: Patagonia, Argentina, Medicinal traditional knowledge, Organoleptic traits

\footnotetext{
* Correspondence: ahladio@gmail.com

${ }^{2}$ INIBIOMA, CONICET- Universidad Nacional del Comahue, Quintral 1250,

Bariloche 8400, Río Negro, Argentina

Full list of author information is available at the end of the article
} 


\section{Background}

Cultural landscapes are intimately linked ecologicalcultural systems, and therefore seen as biocultural phenomena [1]. They are the result of physical, biological, cultural and social diversity, and their structure is delineated by diverse learning and adaptation processes which are put into practice to deal with environmental circumstances [2]. Domestication processes, successional management, efficient use of environmental heterogeneity and other forms of interaction with plant components are examples of how humans shape their environment $[3,4]$. Cultural landscapes are also the result of particular cosmovisions, with symbolic meanings and values which transcend mere usefulness; they signify vital opportunities for nourishment, recreation and healing, among many other functions [5].

With respect to cultural landscapes as a source of therapeutic resources, Sõukand and Kalle [6] propose the concept of 'herbal landscape', referring to the area where the psycho-physical and emotional needs of individuals or social groups are generated and where treatments and prophylactic or healing measures can be taken. According to these authors, limits in terms of space are determined and demarcated as much according to geoclimatic factors as to socio-cultural ones. These limits are not absolute, but flexible, and allow the entry and exit of native and exotic plants, people, knowledge and processes, and are therefore functionally dynamic over time [7].

According to different authors $[6,8]$, perception of the landscape as a domestic, therapeutic and agro-breeding area is found to be strongly influenced by the sensorial qualities of its components, and is established by the particular points of view of the human group which passes through, uses and reproduces it. However, this phenomenon has been little taken into account up to now as a focus of study [8]. It has been proposed that the sensorial appearance of plants, that is, the set of its characteristics of flavor, aroma, color, texture, shape etc., could act as a link between socially developed ideas and the plant world, influencing the people's behavior in terms of group strategies for exploring habitats and the use of resources [9]. This is why sensorial stimuli play an important role in diet selection, medicine, religion, memory, sexuality, the relationship between human groups and their natural environment [9], especially in groups which are closely associated with their natural surroundings [10].

Previous work on this subject in Subantarctic Patagonia indicate that species with highest consensus of use have the most agreement in terms of their characteristic of taste and smell [8]. A specific study of gastrointestinal Patagonian forest plants found that stomach complaints are treated with delicate and sweet plants, that hepatic complaints are generally treated with bitter ones, and that plants used as laxatives are generally spicy [11]. Moreover, we found that the intensity of aroma and/or flavor perceived by people can influence the dosage and method of administration of the herbal preparation $[8,11]$.

On the other hand, studies on environmental perception and the successional management of sub Antarctic forest by Mapuche communities in order to obtain medicinal plants have found that the emic landscape categories seem to be founded on the order given to the universe, relationships between the people and past phenomena, and on current, everyday life $[11,12]$. In addition, the diverse collective interpretations of sensorial signs have been related to the therapeutic and symbolic usefulness of the environmental units and their plants [8].

Knowledge of these aspects in arid zones is still very scarce, particularly in Patagonia. However, it is known that the wild aromatic and sapid plants of arid environments, and the cultural perceptions associated with them, play an important role within traditional medical systems [13-15], possibly, to some extent, in response to their high abundance and visibility in these ecosystems [16].

The rural populations living in arid Patagonia, which are mainly of Mapuche-Tehuelche origin, have developed numerous strategies for the use of natural resources which are often scarce and/or inaccessible due to the inhospitable context $[17,18]$. Among these strategies, the exploration and differential use of the natural surroundings, the ecological transformation of the land through, for example, cultivation, and the protection given to certain plants, are behaviors that demonstrate the strong cultural roots underlying the search and promotion of medicinal species $[19,20]$. For example, the exotic plants with a strong aroma and flavor that are cultivated in homegardens are valuable elements in the pharmacopeias of this region [11]. In relation to this, differential relevance of the domestic environments as providers of medicinal plants has been suggested, such as the peridomestic and the secondary ecosystems [21].

In this work with the Nahuelpan Mapuche-Tehuelche community of the Argentine Patagonian steppe, the cultural landscape as a source of medicinal plants (herbal landscape) are analyzed from an eco-sensorial approach. The sensorial characteristics of these plants and their relation to the perception, classification and use of the environment are investigated.

Our hypotheses were: 1) The cultural landscape associated with medicinal use of its plant resources (herbal landscape) is perceived, classified and named according to different symbolic, ecological and utilitarian criteria which are influenced by its sensorial appearance. 2) The cultural importance of the different units of the herbal landscape has an organoleptic pattern; it depends on the sensorial characteristics, relative importance and symbolic value of the medicinal species they are composed 
of. 3) The plants used for diverse therapeutic purposes have distinctive eco-sensorial features which are perceived and valued by inhabitants. 4) Species with high consensus of use and relative importance, in any environment and use category, have the highest consensus about its organoleptic characteristics (taste and/or aroma).

\section{Materials and methods}

\section{Study area}

Nahuelpan is situated in the north west of Chubut province, Patagonia, Argentina $\left(43^{\circ} \mathrm{S}, 71^{\circ} \mathrm{W}\right)$ (Figure 1). The general climatic characteristics are strong winds and frosts all year round, snow in winter and a dry season in summer. The average annual temperature is $8^{\circ} \mathrm{C}$, with precipitation of approximately $400 \mathrm{~mm}$ annually. Phytogeographically, this area is included in the occidental Patagonian district, Patagonia province [22]. The dominant vegetation is typical grass-bush steppe, with an abundance of graminaceous Poa spp., Pappostipa spp. and Festuca spp., subshrubs of Mulinum spinosum Cav. (Pers.), bushes of Nassauvia glomerulosa (Lag. ex Lindl.) D. Don, N. axillaris (Lag. ex Lindl.) D. Don, Berberis microphylla G. Forst., Adesmia volckmannii Phil., Nardophyllum bryoides (Lam.)
Cabrera, Azorella monantha Clos, Senecio filaginoides DC., and in some sectors Corynabutilon bicolor (Phil. ex K. Schum.) and Schinus roigii Ruiz Leal \& Cabrera. Among the herbaceous plants the most notable are Cerastium arvense L., Oenothera odorata Jack., Arjona tuberosa Cav., Euphorbia collina Phil., Plantago lanceolada L., Acaena pinnatifida Ruiz \& Pav., Calceolaria sp., Rumex acetosella L., among other plants. In areas close to "mallines" (wetlands of glacial origin) the woody plants Discaria spp., Schinus patagonicus (Phil.) I.M. Johnst. ex Cabrera and Baccharis obovata Hook. \& Arn. grow, as well as the herbaceous Mutisia retrorsa Cav. and Taraxacum officinale G. Weber ex F.H. Wigg., among others. In the highest areas with southern exposure, or in the wet gullies, stunted Nothofagus pumilio (Poepp. \& Endl.) Krasser forests grow [22].

\section{The Nahuelpan community}

The Nahuelpan community is composed of 60 people, living in 21 homes. Land ownership is communal, under the category 'indigenous reserve', with the exception of two families who have a title deed to their properties. The area of indigenous reserve is 7500 hectares [23].

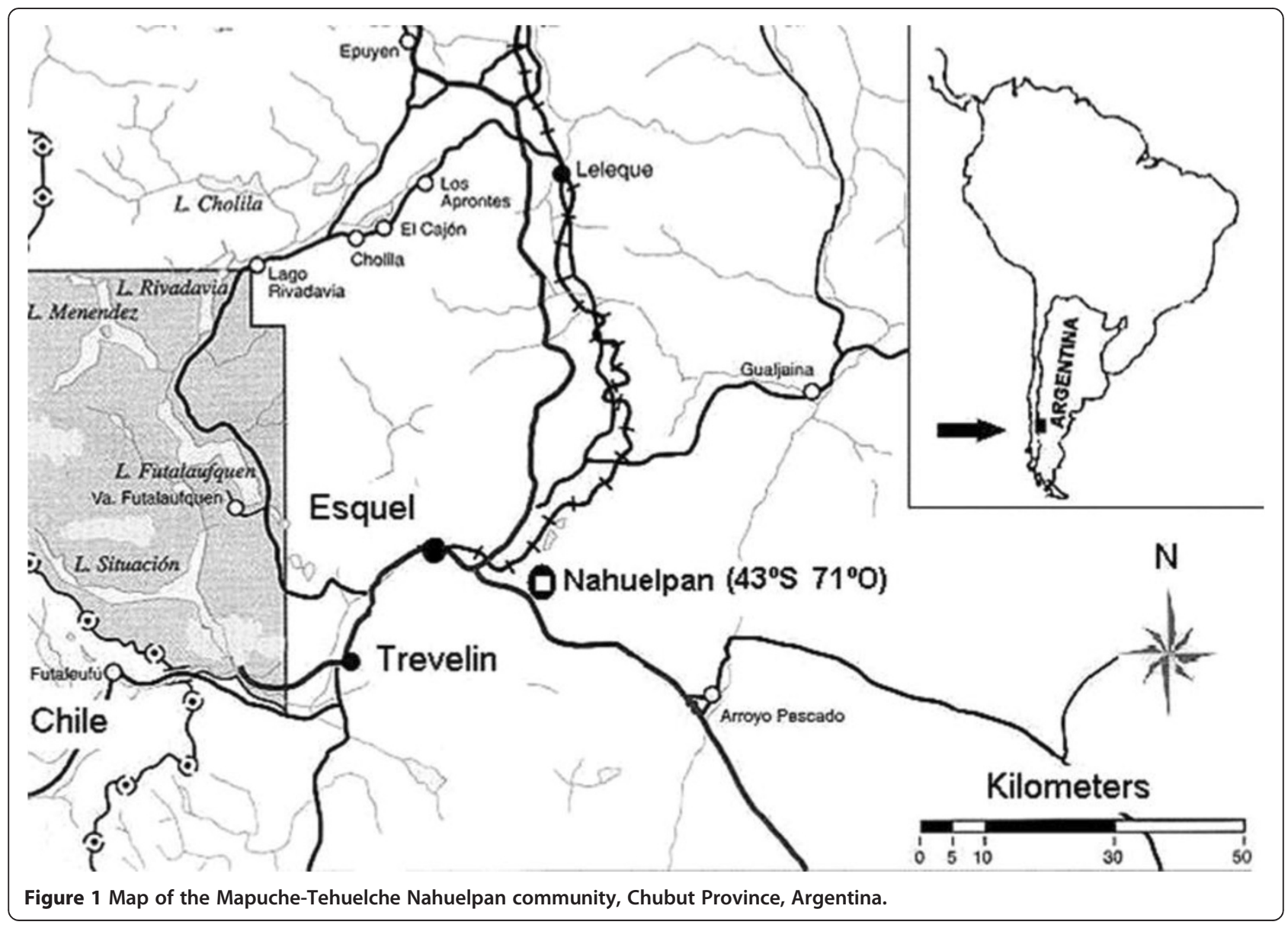


This population comes under the jurisdiction of the municipal government of Esquel (43900 inhabitants, town center $15 \mathrm{~km}$ distant), department Futaleufú. Most inhabitants are Mapuches, and some are Tehuelches. The Mapuche native language is Mapuzungun ('language of the land'), of the Nahuelpan linguistic tribe, but at present only $6 \%$ of the population are active speakers, and they are elderly and bilingual [23]. There are no speakers of the native Tehuelche language.

The principal economic activity is livestock raising, on a large scale, with sheep and goats bred for wool and meat production. The products originating from this activity satisfy only basic subsistence requirements.

One of the stations of the old narrow gauge railway "La Trochita" is found in this locality, and is of recognized value for national and international tourism, thus favoring the sale of knitted garments and regional foods (tortas fritas, jams and home made bread) to the tourists who arrive regularly for a visit.

The main public institution is the primary school, where cultural events take place such as workshops teaching the Mapuche language, veterinary medicine, vegetable gardening, spinning, wool dyeing and knitting, generally organized by extension organizations such as the National Institute of Farming Technology (INTA: Instituto Nacional de Tecnología Agropecuaria) or the Social Farming Program (PSA: Programa Social Agropecuario). Funeral wakes are also held in the school buildings, which function as a community center.

Most of the population dwells close to the school and train station, where the craft shop and a small museum dedicated to the history and indigenous culture of Patagonia are also situated. The remaining dwellings are located between one and fifteen kilometers from this center.

Inhabitants usually move around between houses, or go to the school or Esquel on horseback, on foot or by hitching a lift, since few own a vehicle. There is no public transport in the area. Nevertheless, very frequent visits are made to Esquel since most families have relatives living in this city.

The local medical system can be identified as Creole [24], since therapeutic criteria pertaining to the Mapuche culture [25], coexist with European elements, Christian symbolism, magic practices and the official medicine. The community has no health clinic, but a health worker who resides in the community visits the homes periodically and constitutes a link with the regional hospital in Esquel.

The absence of alternative work, the scarcity of land, which limits the size of the herds, and the lack of water, which restricts the development of agriculture, among other socio economic factors, converge and lead to an accelerated process of migration of the youngest community members, who move mainly to the cities of Esquel,
Trelew $(607 \mathrm{~km})$ and Comodoro Rivadavia $(595 \mathrm{~km})$. Consequently, there are an elevated proportion of adults and elderly in the population.

\section{Collection of ethnobotanical data}

Fieldwork was carried out within the framework of the principles established by the United Nations 1992 Rio de Janeiro Convention on Biological Diversity for the regulation of access to genetic resources and associated knowledge, protection of traditional knowledge and recognition of rights to intellectual property [26]. Authorization for this study was requested from local officials (tribal chief, health worker and headmistress of local school). In addition, informed consent was requested from each interviewee.

Information relating to the cultural landscape experienced by each informant in terms of medicinal plants, knowledge of species and their organoleptic perception was obtained through participant observation, free listing and semi-structured and in-depth interviews, considering that the concept of perception is equivalent to representation, as the externalization of what an individual perceives through all physiological pathways (biological, psychological and cultural senses) [27].

Sampling method consisted of a census of all households in the community. A family of each house was invited to participate in the study. Eighteen families agreed to participate, and one member of each family offered to be interviewed voluntarily. In total, 14 permanent inhabitants and 4 semi-permanent (individuals who live part of the week in Esquel) were interviewed, all representing $85 \%$ of the families.

We use a free listed technique for studied the richness of known and used medicinal species, and its Creole and Mapuzungun names. Through interviews we inquire the methods of preparation and administration of each species, procurement strategies (collection, cultivation, purchase and/or barter), and gathering environments. In the depth analysis of the discourses we approach to the importance of the general chemo-sensorial (aroma and taste) perception involved in the recognition, selection and use of medicinal plants, as well as other items of general interest.

In addition, walks with and without informants were carried out, during which reference material was collected which was placed in the BCR herbarium of the Universidad Nacional del Comahue. The botanical nomenclature used follows Zuloaga et al. [28]. The Mapuzungun nomenclature used follows Díaz Fernández [23].

\section{Data analysis}

Analysis was quali-quantitative, following different methods used in the study of environmental perception to assess worldviews, sensations, values and opinions [27]. 
The nomenclature and biogeographical origin (native or exotic to Patagonia) of the medicinal species was checked by consulting Zuloaga et al. [28]. Total species richness and richness of botanical families was estimated using the total sum of species and families respectively. Use consensus for each species was calculated with the formula: number of informants who cited each species/ total number of informants $(\mathrm{N}=18) \times 100$ [8].

Using qualitative discourse analysis [27], opinions given by the informants regarding the intensity, characteristics and quality of the plant organoleptic sensations recognized were analyzed, and in particular, the significance of the plants and of their sensorial attributes as expressed in Mapuzungun. Following this, the perceptions of aroma and taste were classified according to the informants' (emic) criteria.

The consensus on organoleptic characteristics (OC) was also estimated using their relative frequencies (total number of mentions of the organoleptic characteristic under consideration for the species $i \times 100 /$ total number of informants who cite the species $i$ ) [29].

Following this, the medicinal uses were grouped in etic categories in order to enable quantitative comparisons to be made [25]: gastro-intestinal (GI), respiratory (RE), analgesic-anti-inflammatory (AAI), dermatologicalcosmetic (DC), cultural syndromes (CUS), fever (F), cardio-vascular $(\mathrm{CV})$, obstetric-gynaecological (OG), genito-urinary (GU), and other uses (endocrine, ophthalmic, etc.) (O). The numbers registered were compared between categories with the Chi squared test $(p<0.05)$.

The analysis of the relation between the frequency of cites for each therapeutic use category and the frequency of organoleptic aroma cites was analyzed with the Spearman test $(p<0.05)$.

The plant landscape units which provide medicinal plants were characterized according to informants' precepts [27]. Aromatic and sapid species richness was compared with the richness of non aromatic and non sapid species for each unit, using the binomial test $(p<0.05)$.

In order to compare the cultural importance of the different plant landscape units, the relative importance (RI) of each species was calculated per unit [30], using the following formula: RI = NBS + NP, where NBS is the number of body systems treated by a particular species (NBSS) divided by the number of body systems treated by the most versatile species (NBSVS); NP is the relation between the number of medicinal properties attributed to a given species (NPS) divided by the total number of properties attributed to the most versatile species (NPVS). Following this, in order to obtain weighted frequencies, the RI values were multiplied by the consensus of each species. The maximum value of this index is 200 . The values thus obtained were compared between different landscape units with the Kruskal-Wallis test [8].

\section{Results and discussion}

\section{Herbal landscape and eco-sensoriality: collection sites} and their relation to the organoleptic characteristics of medicinal species

The Nahuelpan herb landscape is heterogeneous and includes areas which have been transformed to some extent, presenting different levels and types of relationship between inhabitants and the physical and natural components. In this landscape most medicinal plants are acquired by collection (78\% of cites), and to a lesser extent by cultivation and harvesting (9\%), and purchase or barter $(13 \%)(p<0.05)$ (Table 1$)$.

Gathering in particular is carried out in areas where the successional management of fodder plants and shrubs is considered a priority. This practice increases the frequency of visits to the different herbal landscape units, enabling verification of the presence of resources and changes in their availability, as well as the perception of their colors, flavors and aromas. Plants resources are collected in quantities suitable for family use alone, principally in zones close to water bodies, which include rivers and waterholes known as "menucos" or "aguadas", and flood meadows or "mallines" of glacial origin (23\%). On some family lands, other management practices have been observed, such as the diversion and/or isolation of these water bodies for the protection of medicinal, edible or fodder species of high cultural value. The importance of these peri-aquatic environments as sources of medicinal resources is likely to be a result of their high levels of biodiversity [31], a characteristic valued by local populations [32].

To a lesser extent, gathering is also carried out on the meseta and plains, known as "pampas" and "lomas secas" $(21.5 \%)$ where the stock animals are grazed for most of the year; and on the stony slopes "pedreros de la cordillera" and "nevados" (a high Andean phytogeographic environment) (18\%). This last mentioned region is visited by inhabitants who still maintain the practice of summer sheep and goat pasturing (around 50\% of family heads). Various studies have reported that this summer pasturing constitutes a form of management that allows pastures to rest, and implies successional herb management, which favors more efficient use of weeds spread by the animals [18].

Another environment worthy of note is the area surrounding the dwellings, described in the interviews as "just around here", and other anthropized sites such as the verges of main roads and country roads, paddocks and corrals (16\%). Taking advantage of these readily available and easily accessible ruderal species $[19,33] \mathrm{im}$ plies a kind of management, due to the link between their dispersion and human activities. Particularly, in this environment, an interesting case is the use of Schinopsis lorentzii (Griseb.) Engl. (red quebracho). This species is 
Table 1 Medicinal species used in the Mapuche-Tehuelche Nahuelpan community

\begin{tabular}{|c|c|c|c|c|c|c|c|c|c|}
\hline Species & Family & Common names & Origin & Unit of the landscape & Medicinal uses & Consensus & RI & Rl $\times$ Consensus & $\begin{array}{l}\text { Sensoria } \\
\text { property }\end{array}$ \\
\hline Gunnera tinctoria (Molina) Mirb. & Gunneraceae & Nalca & $\mathrm{Na}$ & $\begin{array}{l}\text { Water bodies/Purchase/ } \\
\text { barter }\end{array}$ & $\begin{array}{l}\mathrm{Gl}, \mathrm{RE}, \mathrm{CV}, \mathrm{AAl}, \mathrm{CUS}, \mathrm{OG}, \\
\mathrm{GU}\end{array}$ & 83.3 & 1.9 & 156.3 & TA \\
\hline Valeriana carnosa Sm. & Valerianaceae & Namkulawen ${ }^{b}$ & $\mathrm{Na}$ & Stony slopes & $\begin{array}{l}\mathrm{Gl}, \mathrm{RE}, \mathrm{CV}, \mathrm{AAl}, \mathrm{DC}, \mathrm{CUS}, \\
\mathrm{O}, \mathrm{GU}\end{array}$ & 66.7 & 1.9 & 127.3 & TA, SM \\
\hline Artemisia absinthium L. & Asteraceae & $\begin{array}{l}\text { Ajenco, ajenco plateado, } \\
\text { ajenco blanco }\end{array}$ & Ex & Anthropized areas & $\mathrm{Gl}, \mathrm{RE}, \mathrm{AAl}, \mathrm{DC}, \mathrm{CUS}, \mathrm{F}$ & 72.2 & 1.7 & 119.8 & TA, SM \\
\hline Adesmia boronioides Hook. f. & Fabaceae & Paramela & $\mathrm{Na}$ & Stony slopes & $\mathrm{Gl}, \mathrm{RE}, \mathrm{AAl}, \mathrm{DC}, \mathrm{CUS}, \mathrm{O}, \mathrm{GU}$ & 66.7 & 1.7 & 112.9 & TA, SM \\
\hline Fabiana imbricata Ruiz \& Pav. & Solanaceae & Palo piche & $\mathrm{Na}$ & Water bodies & $\begin{array}{l}\text { Gl, RE, CV, AAl, DC, CUS, } \\
\text { OG, GU }\end{array}$ & 72.2 & 1.5 & 111.6 & $\mathrm{TA}, \mathrm{SM}$ \\
\hline Neuropogon sp. ${ }^{a}$ & Parmeliaceae & Barba de piedra & $\mathrm{Na}$ & Stony slopes & $\mathrm{Gl}, \mathrm{RE}, \mathrm{CV}, \mathrm{CUS}, \mathrm{OG}, \mathrm{GU}$ & 61.1 & 1.5 & 90.3 & TA \\
\hline Equisetum bogotense Kunth & Equisetaceae & Limpia plata & $\mathrm{Na}$ & Water bodies & $\begin{array}{l}\text { Gl, RE, CV, AAl, DC, CUS, } \\
F, G U\end{array}$ & 44.4 & 1.8 & 80.8 & NO \\
\hline Erodium cicutarium (L.) L'Hér. ex Aiton & Geraniaceae & Alfilerillo & Ex & Anthropized areas & $\mathrm{Gl}, \mathrm{RE}, \mathrm{CV}, \mathrm{DC}, \mathrm{CUS}, \mathrm{F}$ & 50 & 1.6 & 78.4 & NO \\
\hline Buddleja globosa Hope & Buddlejaceae & Pañil ${ }^{b}$ & $\mathrm{Na}$ & Purchase/barter & $\mathrm{Gl}, \mathrm{AAl}, \mathrm{DC}, \mathrm{CUS}, \mathrm{OG}, \mathrm{O}$ & 38.9 & 1.8 & 68.1 & $\mathrm{TA}, \mathrm{SM}$ \\
\hline Acantholippia seriphioides (A. Gray) Moldenke & Verbenaceae & Tomillo del campo & $\mathrm{Na}$ & Dry plates & $\mathrm{Gl}, \mathrm{RE}, \mathrm{CV}, \mathrm{CUS}, \mathrm{GU}$ & 44.4 & 1.3 & 56.1 & $\mathrm{TA}, \mathrm{SM}$ \\
\hline Marrubium vulgare $\mathrm{L}$. & Lamiaceae & Malva rubia & Ex & Anthropized areas & $R E, F, O$ & 61.1 & 0.8 & 50.7 & TA \\
\hline Matricaria recutita $\mathrm{L}$. & Asteraceae & Manzanilla & Ex & Purchase & $\mathrm{Gl}, \mathrm{AAl}, \mathrm{CUS}, \mathrm{O}$ & 38.9 & 1.2 & 47.7 & $\mathrm{TA}, \mathrm{SM}$ \\
\hline Baccharis sagittalis (Less.) DC. & Asteraceae & Carqueja & $\mathrm{Na}$ & Water bodies & $\mathrm{Gl}, \mathrm{AAl}$ & 83.3 & 0.6 & 51.1 & TA, SM \\
\hline Grindelia chiloensis (Cornel.) Cabrera & Asteraceae & Botón de oro & $\mathrm{Na}$ & Dry plates & $\mathrm{RE}, \mathrm{AAl}, \mathrm{DC}, \mathrm{CUS}, \mathrm{F}, \mathrm{O}$ & 38.9 & 1.1 & 43.3 & SM \\
\hline Anarthrophyllum sp. & Fabaceae & Nenew $^{\mathrm{b}}$ macho & $\mathrm{Na}$ & Dry plates & $\mathrm{RE}, \mathrm{OG}, \mathrm{O}$ & 50 & 0.7 & 36.9 & $\mathrm{TA}, \mathrm{SM}$ \\
\hline Apium prostratum Labill. & Apiaceae & Apio del campo & $\mathrm{Na}$ & Stony slopes & $\mathrm{RE}, \mathrm{AAl}, \mathrm{F}, \mathrm{O}$ & 44.4 & 0.9 & 38.4 & $\mathrm{TA}, \mathrm{SM}$ \\
\hline Ribes cucullatum Hook. \& Arn. & Grossulariaceae & Zarzaparrilla & $\mathrm{Na}$ & Water bodies & CV, DC, CUS, O & 44.4 & 0.9 & 38.4 & SA, AR \\
\hline Acaena splendens Hook. \& Arn. & Rosaceae & Cesped caballo & $\mathrm{Na}$ & Dry plates & $\mathrm{RE}, \mathrm{AAl}, \mathrm{OG}, \mathrm{GU}$ & 33.3 & 0.9 & 28.8 & TA \\
\hline Oxalis sp. & Oxalidaceae & Culle verde & $\mathrm{Na}$ & Stony slopes & $\mathrm{RE}, \mathrm{OG}, \mathrm{F}$ & 38.9 & 0.8 & 32.3 & TA \\
\hline Ruta chalepensis $L$. & Rutaceae & Ruda & Ex & Cultivated areas & $\mathrm{Gl}, \mathrm{AAl}, \mathrm{CUS}$ & 27.8 & 0.9 & 25.6 & TA, SM \\
\hline Tanacetum vulgare $\mathrm{L}$. & Asteraceae & $\begin{array}{l}\text { Ajenco amarillo, ajenco, } \\
\text { ajenco verde }\end{array}$ & Ex & Cultivated areas & $\mathrm{Gl}, \mathrm{RE}, \mathrm{AAl}, \mathrm{F}$ & 27.8 & 1.0 & 29.0 & TA, SM \\
\hline Sedum telephium L. & Crassulaceae & Bálsamo & Ex & Cultivated areas & $A A I, D C$ & 33.3 & 0.6 & 20.5 & NO \\
\hline Solanum crispum Ruiz \& Pav. & Solanaceae & Natre & $\mathrm{Na}$ & Purchase/barter & $R E, F$ & 44.4 & 0.5 & 23.2 & TA \\
\hline Clinopodium darwinii (Benth.) Kuntze & Lamiaceae & Té pampa, té de campo & $\mathrm{Na}$ & Stony slopes & $\mathrm{Gl}, \mathrm{GU}$ & 55.6 & 0.4 & 24.0 & $\mathrm{TA}, \mathrm{SM}$ \\
\hline Urtica urens $\mathrm{L}$. & Urticaceae & Ortiga & Ex & Anthropized areas & CV, AAI, DC, CUS, OG & 16.7 & 1.2 & 19.5 & $\mathrm{TA}, \mathrm{SM}$ \\
\hline Baccharis obovata Hook. \& Arn. & Asteraceae & Wawtru ${ }^{b}$ & $\mathrm{Na}$ & Water bodies & $A A I, D C$ & 38.9 & 0.5 & 20.3 & TA, SM \\
\hline Allium cepa L. & Alliaceae & Cebolla & Ex & Purchase & RE, CUS, F & 16.7 & 0.8 & 13.8 & TA, SM \\
\hline Oxalis adenophylla Gillies ex Hook. \& Arn. & Oxalidaceae & Culle colorado & $\mathrm{Na}$ & Stony slopes & $\mathrm{RE}, \mathrm{DC}, \mathrm{OG}, \mathrm{F}$ & 22.2 & 0.9 & 19.2 & TA \\
\hline
\end{tabular}


Table 1 Medicinal species used in the Mapuche-Tehuelche Nahuelpan community (Continued)

\begin{tabular}{|c|c|c|c|c|c|c|c|c|c|}
\hline Stillingia patagonica (Speg.) Pax \& K. Hoffm. & Euphorbiaceae & Trintri lawen ${ }^{\mathrm{b}}$, mata perro & $\mathrm{Na}$ & Dry plates & $\mathrm{Gl}, \mathrm{AAl}, \mathrm{DC}$ & 16.7 & 0.8 & 13.8 & TA \\
\hline Armeria maritima (Mill.) Willd. & Plumbaginaceae & Piuke lawen ${ }^{b}$ & $\mathrm{Na}$ & Dry plates & $\mathrm{Gl}, \mathrm{CV}, \mathrm{AAl}$ & 22.2 & 0.7 & 16.4 & TA, SM \\
\hline $\begin{array}{l}\text { Dysphania ambrosioides (L.) Mosyakin \& } \\
\text { Clemants }\end{array}$ & Chenopodiaceae & Paico & $\mathrm{Na}$ & Anthropized areas & Gl & 38.9 & 0.4 & 15.5 & TA, SM \\
\hline Mentha pulegium L. & Lamiaceae & Poleo & Ex & Water bodies & $\mathrm{Gl}$ & 38.9 & 0.3 & 11.9 & TA, SM \\
\hline Chuquiraga avellanedae Lorentz & Asteraceae & $\begin{array}{l}\text { Trayaw }{ }^{b} \text {, pincha bola, } \\
\text { quilembay }\end{array}$ & $\mathrm{Na}$ & Dry plates & RE & 33.3 & 0.5 & 16.3 & TA \\
\hline Gossyphiantus lanuginosus (Poir.) Hook. f.? & Amaranthaceae & Yerba del pollo & Ex & Purchase & Gl & 22.2 & 0.5 & 10.9 & TA, SM \\
\hline Limonium brasiliense (Boiss.) Kuntze & Plumbaginaceae & Guaycurú & $\mathrm{Na}$ & Dry plates & Gl, RE, AAl, CUS & 16.7 & 0.9 & 14.4 & TA \\
\hline Maihuenia patagonica (Phil.) Britton \& Rose & Cactaceae & Chupasangre, tuna & $\mathrm{Na}$ & Dry plates & $C V, A A I, D C$ & 16.7 & 0.6 & 10.8 & NO \\
\hline Melissa officinalis L. & Lamiaceae & Torongil, melisa & Ex & Cultivated areas & $\mathrm{Gl}, \mathrm{CV}, \mathrm{CUS}$ & 27.8 & 0.6 & 18.0 & TA, SM \\
\hline Mulinum spinosum (Cav.) Pers. & Apiaceae & Nenew $^{\mathrm{b}}$ & $\mathrm{Na}$ & Dry plates & RE, AAI, O & 16.7 & 0.6 & 10.8 & TA, SM \\
\hline Sambucus nigra L. & Adoxaceae & Sauco & Ex & Cultivated areas & $R E, A A I, F$ & 27.8 & 0.6 & 18.0 & TA \\
\hline Dysphania multifida L. & Chenopodiaceae & Paico & Ex & Anthropized areas & $\mathrm{Gl}, \mathrm{RE}$ & 22.2 & 0.5 & 11.6 & TA, SM \\
\hline Plantago lanceolata $\mathrm{L}$. & Plantaginaceae & Siete venas, siete velos & Ex & Anthropized areas & $A A I, D C$ & 22.2 & 0.5 & 11.6 & NO \\
\hline Centaurium cachanlahuen (Molina) B.L. Rob. & Gentianaceae & $\begin{array}{l}\text { Kachanlawenb, } \\
\text { cachanlagua }\end{array}$ & $\mathrm{Na}$ & Stony slopes & $R E, F$ & 22.2 & 0.4 & 9.6 & TA \\
\hline Corynabutilon bicolor (Phil. ex K. Schum.) Kearney & Malvaceae & Monte moro & $\mathrm{Na}$ & Dry plates & RE, CUS, F & 16.7 & 0.7 & 12.3 & NO \\
\hline Eucalyptus sp. & Myrtaceae & Eucalito & Ex & Purchase/barter & RE, CUS & 16.7 & 0.5 & 8.7 & TA, SM \\
\hline Linum usitatissimum L. & Linaceae & Linaza, lino & Ex & Purchase & $O G, F$ & 16.7 & 0.5 & 8.7 & TA \\
\hline Rosmarinus officinalis $\mathrm{L}$. & Lamiaceae & Romero & Ex & Purchase & Gl, AAl, CUS, O & 11.1 & 0.9 & 9.6 & TA, SM \\
\hline Rumex crispus $L$. & Polygonaceae & Romaza, lengua de vaca & Ex & Water bodies & $\mathrm{Gl}, \mathrm{F}$ & 22.2 & 0.4 & 9.6 & NO \\
\hline $\begin{array}{l}\text { Tetraglochin alatum (Gillies ex Hook. \& Arn.) } \\
\text { Kuntze }\end{array}$ & Rosaceae & Yerba de la perdiz & $\mathrm{Na}$ & Dry plates & $\mathrm{Gl}, \mathrm{OG}, \mathrm{GU}$ & 16.7 & 0.7 & 12.3 & TA \\
\hline Artemisia abrotanum $\mathrm{L}$. & Asteraceae & Buen viejo, éter & Ex & Anthropized areas & $\mathrm{Gl}$ & 27.8 & 0.3 & 8.5 & TA, SM \\
\hline Azorella monantha Clos & Apiaceae & Leña de piedra & $\mathrm{Na}$ & Dry plates & $\mathrm{Gl}$ & 27.8 & 0.3 & 8.5 & TA, SM \\
\hline Larrea sp. & Zygophyllaceae & Jarilla & $\mathrm{Na}$ & Dry plates & $\mathrm{AAl}, \mathrm{OG}$ & 11.1 & 0.4 & 4.8 & TA, SM \\
\hline Mentha spicata L. & Lamiaceae & Menta, yerba buena & Ex & Water bodies & $\mathrm{Gl}, \mathrm{RE}$ & 27.8 & 0.4 & 12.0 & TA, SM \\
\hline Mentha aquatica $\mathrm{L}$. & Lamiaceae & $\begin{array}{l}\text { Menta canela, menta, } \\
\text { menta negra }\end{array}$ & Ex & Cultivated areas & Gl & 22.2 & 0.2 & 4.8 & TA, SM \\
\hline Polygonum aviculare L. & Polygonaceae & Sanguinaria & Ex & Anthropized areas & $\mathrm{CV}, \mathrm{GU}$ & 11.1 & 0.4 & 4.8 & TA \\
\hline Schinus patagonicus (Phil.) I.M. Johnst. ex Cabrera & Anacardiaceae & Laura & $\mathrm{Na}$ & Water bodies & RE, CUS & 11.1 & 0.4 & 4.8 & TA, SM \\
\hline Allium sativum $\mathrm{L}$. & Alliaceae & Ajo & Ex & Purchase/barter & $\mathrm{Gl}, \mathrm{AAl}$ & 5.6 & 0.5 & 2.9 & TA, SM \\
\hline Plantago sp. & Plantaginaceae & Siete venas & Ex & Anthropized areas & $\mathrm{DC}, \mathrm{O}$ & 5.6 & 0.3 & 1.9 & NO \\
\hline Schinus roigii Ruiz Leal \& Cabrera & Anacardiaceae & Molle & $\mathrm{Na}$ & Water bodies & RE, DC, CUS & 11.1 & 0.7 & 8.2 & TA \\
\hline
\end{tabular}


Table 1 Medicinal species used in the Mapuche-Tehuelche Nahuelpan community (Continued)

\begin{tabular}{|c|c|c|c|c|c|c|c|c|c|}
\hline$\overline{8^{c}}$ & $?$ & Mata dolor & $?$ & Dry plates & RE, AAl & 16.7 & 0.4 & 7.2 & $\overline{T A}$ \\
\hline $11^{c}$ & $?$ & $?$ & $?$ & Stony slopes & $\mathrm{Gl}, \mathrm{RE}, \mathrm{AAl}$ & 5.6 & 0.6 & 3.6 & TA \\
\hline Malva sylvetris $\mathrm{L}$. & Malvaceae & Malva & Ex & Anthropized areas & $\mathrm{Gl}, \mathrm{O}, \mathrm{GU}$ & 11.1 & 0.6 & 7.2 & NO \\
\hline Oenothera odorata Jacq. & Onagraceae & Yerba de san Juan & $\mathrm{Na}$ & Dry plates & $?$ & 16.7 & 0.2 & 3.6 & TA \\
\hline Origanum vulgare L. & Lamiaceae & Orégano & Ex & Purchase & $\mathrm{RE}, \mathrm{OG}, \mathrm{O}$ & 11.1 & 0.6 & 7.2 & $\mathrm{TA}, \mathrm{SM}$ \\
\hline Senecio sp. 1 & Asteraceae & Charkaw $^{\mathrm{b}}$ & $\mathrm{Na}$ & Dry plates & $\mathrm{RE}, \mathrm{AAl}$ & 16.7 & 0.4 & 7.2 & TA, SM \\
\hline Viola maculata Cav. & Violaceae & Oreja de ratón & $\mathrm{Na}$ & Dry plates & $\mathrm{Gl}$ & 11.1 & 0.5 & 5.4 & NO \\
\hline Lycium chilense Miers ex Bertero & Solanaceae & Monte negro & $\mathrm{Na}$ & Dry plates & OG & 11.1 & 0.4 & 4.4 & TA \\
\hline Mutisia retrorsa Cav. & Asteraceae & Redadera & $\mathrm{Na}$ & Water bodies & RE & 5.6 & 0.3 & 1.7 & $\mathrm{TA}, \mathrm{SM}$ \\
\hline $9^{c}$ & $?$ & Monte verde & $?$ & Stony slopes & $\mathrm{Gl}, \mathrm{CUS}$ & 5.6 & 0.4 & 2.4 & NO \\
\hline $10^{c}$ & $?$ & $?$ & $?$ & Dry plates & $\mathrm{Gl}$ & 11.1 & 0.2 & 2.4 & TA, SM \\
\hline $13^{c}$ & $?$ & $?$ & $?$ & Purchase/barter & Gl, CUS & 5.6 & 0.4 & 2.4 & NO \\
\hline $4^{c}$ & Poaceae & Pasto de perro & $?$ & Anthropized areas & $\mathrm{Gl}, \mathrm{F}$ & 5.6 & 0.4 & 2.4 & NO \\
\hline $6^{c}$ & $?$ & Flor de piedra & $?$ & Stony slopes & $\mathrm{Gl}, \mathrm{CUS}$ & 5.6 & 0.4 & 2.4 & NO \\
\hline Discaria sp. & Rhamnaceae & Chakay ${ }^{b}$ & $\mathrm{Na}$ & Water bodies & $\mathrm{Gl}, \mathrm{RE}$ & 5.6 & 0.4 & 2.4 & NO \\
\hline Gamocarpha selliana Reiche & Calyceraceae & Pata de williñ ${ }^{b}$ & $\mathrm{Na}$ & Water bodies & $\mathrm{Gl}, \mathrm{CUS}$ & 5.6 & 0.4 & 2.4 & NO \\
\hline Mentha suaveolens Ehrh. & Lamiaceae & Menta chilena & Ex & Cultivated areas & $\mathrm{Gl}, \mathrm{DC}$ & 5.6 & 0.4 & 2.4 & TA, SM \\
\hline Nardophyllum bryoides (Lam.) Cabrera & Asteraceae & Siete camisas & $\mathrm{Na}$ & Dry plates & $\mathrm{Gl}$ & 11.1 & 0.2 & 2.4 & TA, SM \\
\hline Nassauvia glomerulosa (Lag. ex Lindl.) D. Don & Asteraceae & $\begin{array}{l}\text { Uña de gato, cola } \\
\text { de piche }\end{array}$ & $\mathrm{Na}$ & Dry plates & O & 11.1 & 0.2 & 2.4 & NO \\
\hline Oryza sativa L. & Poaceae & Arroz & Ex & Purchase & $\mathrm{Gl}$ & 11.1 & 0.2 & 2.4 & TA \\
\hline Peumus boldus Molina & Monimiaceae & Boldo & Ex & Purchase/barter & $\mathrm{Gl}$ & 11.1 & 0.2 & 2.4 & $\mathrm{TA}, \mathrm{SM}$ \\
\hline Quillaja saponaria Molina & Quillajaceae & Palo jabón, jabón de palo & Ex & Purchase & CV & 11.1 & 0.2 & 2.4 & TA, SM \\
\hline Rosa rubiginosa $\mathrm{L}$. & Rosaceae & Rosa mosqueta & Ex & Anthropized areas & $\mathrm{RE}, \mathrm{O}$ & 5.6 & 0.4 & 2.4 & TA \\
\hline Sanicula graveolens Poepp. ex DC. & Apiaceae & $\begin{array}{l}\text { Cilantro de campo, } \\
\text { cilantro silvestre }\end{array}$ & $\mathrm{Na}$ & Water bodies & $\mathrm{Gl}$ & 11.1 & 0.2 & 2.4 & $\mathrm{TA}, \mathrm{SM}$ \\
\hline Senecio sp. 2 & Asteraceae? & Paco & $\mathrm{Na} ?$ & Stony slopes & $D C, F$ & 5.6 & 0.4 & 2.4 & SM \\
\hline Tilia sp. & Tiliaceae & Tilo & Ex & Purchase & Gl, CUS & 5.6 & 0.4 & 2.4 & TA \\
\hline Valeriana clarionifolia Phil. & Valerianaceae & Namkulawen ${ }^{\text {b }}$ & $\mathrm{Na}$ & Stony slopes & $\mathrm{CV}, \mathrm{AAI}$ & 5.6 & 0.4 & 2.4 & TA, SM \\
\hline Verbascum thapsus L. & Scrophulariaceae & Tabaco, tabaco de turco & Ex & Anthropized areas & $?$ & 11.1 & 0.2 & 2.4 & SM \\
\hline Populus nigra L. & Salicaceae & Álamo & Ex & Cultivated areas & $\mathrm{Gl}$ & 5.6 & 0.4 & 2.2 & NO \\
\hline $1^{c}$ & $?$ & Füre lawen ${ }^{b}$ & $?$ & Dry plates & RE & 5.6 & 0.2 & 1.2 & TA, SM \\
\hline $12^{c}$ & $?$ & ? & $?$ & Dry plates & DC & 5.6 & 0.2 & 1.2 & NO \\
\hline $2^{c}$ & Asteraceae & Cardo & Ex & Anthropized areas & 0 & 5.6 & 0.2 & 1.2 & TA \\
\hline
\end{tabular}


Table 1 Medicinal species used in the Mapuche-Tehuelche Nahuelpan community (Continued)

\begin{tabular}{|c|c|c|c|c|c|c|c|c|c|}
\hline $3^{c}$ & $?$ & $?$ & $?$ & Dry plates & $\mathrm{Gl}$ & 5.6 & 0.2 & 1.2 & $\mathrm{NO}$ \\
\hline $5^{c}$ & $?$ & Poñí cachob & $?$ & Dry plates & DC & 5.6 & 0.2 & 1.2 & NO \\
\hline $7^{c}$ & Juncaceae & Unquillo & $?$ & Water bodies & DC & 5.6 & 0.2 & 1.2 & NO \\
\hline Aloe sp. & Xanthorrhoeaceae & Aloe & Ex & Cultivated areas & GU & 5.6 & 0.2 & 1.2 & NO \\
\hline Aristotelia chilensis (Molina) Stuntz & Elaeocarpaceae & Make ${ }^{b}$ & $\mathrm{Na}$ & Barter & $\mathrm{Gl}$ & 5.6 & 0.2 & 1.2 & NO \\
\hline Beta vulgaris $\mathrm{L}$. & Chenopodiaceae & Acelga & Ex & Cultivated areas & $\mathrm{O}$ & 5.6 & 0.2 & 1.2 & TA \\
\hline Calceolaria uniflora Lam. & Calceolariaceae & $?$ & $\mathrm{Na}$ & Dry plates & RE & 5.6 & 0.2 & 1.2 & NO \\
\hline Camellia sinensis (L.) Kuntze & Theaceae & Té común & Ex & Purchase & O & 5.6 & 0.2 & 1.2 & TA, SM \\
\hline Conium maculatum L. & Apiaceae & Cicuta & Ex & Anthropized areas & $\mathrm{O}$ & 5.6 & 0.2 & 1.2 & SM \\
\hline Euphorbia collina Phil. & Euphorbiaceae & Pichoga & $\mathrm{Na}$ & Dry plates & $\mathrm{Gl}$ & 5.6 & 0.2 & 1.2 & NO \\
\hline Ilex paraguariensis A. St. -Hil. & Aquifoliaceae & Yerba mate & Ex & Purchase/barter & OG & 5.6 & 0.2 & 1.2 & TA \\
\hline Iris sp. & Iridaceae & Lirio blanco & Ex & Cultivated areas & CUS & 5.6 & 0.2 & 1.2 & NO \\
\hline Laurus nobilis L. & Lauraceae & Laurel & Ex & Purchase & AAI, CUS & 5.6 & 0.2 & 1.2 & TA, SM \\
\hline Lomatia hirsuta (Lam.) Diels & Proteaceae & Radal & $\mathrm{Na}$ & Stony slopes & AAl & 5.6 & 0.2 & 1.2 & NO \\
\hline Maytenus boaria Molina & Celastraceae & Maitén & $\mathrm{Na}$ & Barter & CUS & 5.6 & 0.2 & 1.2 & NO \\
\hline Mentha sp. & Lamiaceae & Pepermina & Ex & Cultivated areas & $\mathrm{Gl}$ & 5.6 & 0.2 & 1.2 & TA \\
\hline Mutisia decurrens Cav. & Asteraceae & Redadera & $\mathrm{Na}$ & Water bodies & $?$ & 5.6 & 0.2 & 1.2 & TA \\
\hline Nasturtium officinale W. T. Aiton & Brassicaceae & Berro & Ex & Water bodies & $\mathrm{Gl}$ & 5.6 & 0.2 & 1.2 & TA \\
\hline Potentilla chiloensis (L.) Mabb. & Rosaceae & Frutilla & $\mathrm{Na}$ & Water bodies & $\mathrm{O}$ & 5.6 & 0.2 & 1.2 & TA \\
\hline Rhodophiala mendocina (Phil.) Ravenna? & Amaryllidaceae & Juanita & $\mathrm{Na}$ & Dry plates & OG & 5.6 & 0.2 & 1.2 & TA \\
\hline Salvia officinalis L. & Lamiaceae & Salvia & Ex & Cultivated areas & OG & 5.6 & 0.2 & 1.2 & TA, SM \\
\hline Saponaria officinalis L. & Caryophyllaceae & $?$ & Ex & Water bodies & DC & 5.6 & 0.2 & 1.2 & TA, SM \\
\hline Schinopsis lorentzii (Griseb.) Engl. & Anacardiaceae & Quebracho colorado & Ex & Purchase/barter & $\mathrm{AAl}$ & 5.6 & 0.2 & 1.2 & NO \\
\hline Solanum sp. & Solanaceae & Tomatito & $\mathrm{Na}$ & Anthropized areas & DC & 5.6 & 0.2 & 1.2 & TA, SM \\
\hline Tanacetum balsamita L. & Asteraceae & Menta & Ex & Cultivated areas & $?$ & 5.6 & 0.2 & 1.2 & TA, SM \\
\hline Taraxacum officinale G. Weber ex F.H. Wigg & Asteraceae & Chicoria & Ex & Water bodies & $\mathrm{O}$ & 5.6 & 0.2 & 1.2 & TA \\
\hline Tristagma patagonicum (Baker) Traub & Alliaceae & Cebolla de campo & $\mathrm{Na}$ & Dry plates & $?$ & 5.6 & 0.2 & 1.2 & TA \\
\hline Triticum aestivum L. & Poaceae & Trigo & Ex & Purchase/barter & $\mathrm{Gl}$ & 5.6 & 0.2 & 1.2 & TA \\
\hline Tropaeolum incisum (Speg.) Sparre & Tropaeolaceae & $?$ & $\mathrm{Na}$ & Stony slopes & RE & 5.6 & 0.2 & 1.2 & SM \\
\hline Urtica magellanica Poir. & Urticaceae & Ortiga & $\mathrm{Na}$ & Stony slopes & ? & 5.6 & 0.2 & 1.2 & NO \\
\hline
\end{tabular}

aLichen.
bMapuzungun name.

'Undetermined species.

References: biogeographic origin: $\mathrm{Ex}=$ exotic, $\mathrm{Na}=$ native; medicinal uses: $\mathrm{Gl}=$ gastro-intestinal, $\mathrm{RE}=$ respiratory, $\mathrm{AAl}=$ analgesic-anti-inflammatory, $\mathrm{DC}=$ dermatological-cosmetic, $\mathrm{CUS}=\mathrm{cultural}$ syndromes, $\mathrm{F}=$ feber, $\mathrm{CV}=$ cardio-vascular, $\mathrm{OG}=$ obstetric-gynaecological, $\mathrm{GU}=$ genito-urinary, and $\mathrm{O}=$ other uses; $\mathrm{RI}=$ relative importance; sensorial property: $\mathrm{TA}=$ taste, $\mathrm{SM}=$ smell, $\mathrm{NO}=\mathrm{no}$ taste-no smell. 
native to the Chaco region of NE Argentina, and is sold in health food shops and pharmacies in Argentina due to its well-known anti-diarrhoea, emollient, antiseptic and tissue healing powers [34]. Nevertheless, in Nahuelpan this species is obtained mainly by gathering, in this case by the scraping of fence posts or railway track sleepers made of this wood. Thus, the inhabitants take advantage of the cultural landscape in multiple, original ways, according to local availability.

With regard to the eco-sensoriality of these herbal landscape units, it was found that apart from in the "pampas" and "lomas secas", most medicinal plants belonging to remaining environments are basically defined, characterized and valued according to their taste (binomial test, $p<0.05)$. In contrast, with respect to their aromaticity, only in the "pedreros" and "nevados de la cordillera" did aroma figure as a differential trait in most of the plants (binomial test, $p<0.05)$.

However, these units are not isolated and present a certain amount of spatial continuity, not only with respect to ecological gradient but also to the many interconnections generated from the network of roads and tracks established and used by the inhabitants themselves and their domestic animals. Because of this, their limits are not clear-cut, but rather diffuse and porous [7]. According to ethnobotanical approach the physical spaces of cultural landscape are scenarios that reflect an intricate network of people, places and resources over time, so that their boundaries are necessarily dynamic in response to different needs, ideas and cultural practices [2]. In particular, these paths often guide the movements of the shepherds and their flocks, having been tread and re-tread in response to the differential availability of pastures in different years, and the establishment or abandonment of dwellings. These interconnectors themselves favor the exchange of medicinal plants among neighbors, as well as activities associated with other products such as sheep's fleece, tools, food, etc.

\section{Barter and purchase and their relation to the herbal landscape}

The social and economic activity of bartering fulfills an important role in the recreation of the Nahuelpan plant landscape. This practice favors the periodic interaction of families within the community and with others from neighboring communities, or rather, with workers who return from the sheep shearing operations on other Patagonian ranches.

By means of barter, other species from distant environments, which are mostly aromatic and sapid, are added to the repertory of plants used (binomial test, $p<0.05$; Table 1). Among these, worthy of special mention are Larrea sp. ("jarilla") native to the Patagonian Monte, Solanum crispum Ruiz et Pav.("natre") native to sub
Antarctic forests, and Eucalyptus sp. ("eucalito"), an exotic species native to Australia, planted widely along the Atlantic coast that is commonly gather by rural workers in the ranches.

Bartering is complemented with purchasing, an additional strategy which allows the acquisition of fragmented, packaged or loose medicinal resources in grocer's, health food shops and pharmacies in the town of Esquel, or from travelling salespeople. Some authors suppose that this practice could indicate disuse of gathering, or even processes of erosion of traditional knowledge [35]. However, in this case study, purchase as a supply strategy indicates processes leading to the expansion and/or diversification of the therapeutic cultural landscape [6]. In this sense, the incorporation of resources exogenous to Nahuelpan could be associated with an adaptive strategy to deal with a serious environmental circumstance, such as the growing regional process of desertification which limits the availability of some of the repertory of plants the people need (e.g. Matricaria recutita L. and Gunnera tinctorea (Molina) Mirb.).

All the plants purchased are defined and characterized by the presence of flavor and aroma (binomial test, $p<0.05)$.

\section{Homegardens, pot plants and the herbal landscape}

The herbal landscape also includes the homegardens constructed by inhabitants, modifying the appearance of the location, and constituting food and medicinal plant supply sites. These plant sources are frequently situated in peridomestic areas. Using this strategy, fourteen species of exotic medicinal species (e.g. Melissa officinalis L., Mentha spp., Beta vulgaris L., Salvia officinalis L. and Tanacetum balsamita L.) are harvested, which is a low number for the inhabitants, and a consequence of a lack of irrigation water, although the progressive loss of ancestral domestic horticultural practices could also be a cause [20]. The majority of these plants are for alimentary use, and originated from packet seed provided by INTA.

In these cultivation sites preexisting native species in the homegardens are tolerated and/or protected, coexisting with the cultivated ones, as is the case of the shrubs Corynabutilon bicolor (Phil. ex K. Schum.) Kearney and Fabiana imbricata Ruiz et Pav., which are also valued as hedges, for protection and shelter from the wind and sun, and refuge for farm animals. Following [5,36], tolerance is a common management practice here so which these plants are left deliberately standing when vegetation is disturbed; in addition, people make actions of protection favoring permanence of these plants through special care (pruning, elimination of weeds, etc). However, no evidence was found in homegardens of other management practices with this native medicinal plants, such as transplanting or planting seed or propagules from wild areas. 
Other species such as Populus spp., Sambucus nigra L., Tanacetum vulgare L. and Iris spp., are cultivated principally for their ornamental value and as shelter from the wind, and occasionally used for medicinal purposes (Table 1). The fact that these are multifunctional species seems to be a very important selection criterion in rural communities. It has been found that in hedge species, their additional alimentary and/or medicinal value constitutes a notable trait in rural populations of Patagonia [37,38]. However, there are also other medicinal species used which are important due to their uniqueness and effectiveness, such as Salvia officinalis L. for obstetric-gynaecological diseases and Mentha aquatica L. for gastro-intestinal pains.

Three exotic species are cultivated in pots: Sedum telephium L., Ruta chalepensis L. (both outdoors) and Aloe sp. (indoors), which are, in general, plants valued for their therapeutic and symbolic prestige, whose use extends to other Mapuche communities in the region [25]. These are plants which also adorn dwellings, but their principal symbolic function is to act as protectors or defensive elements and purifiers of the domestic environment. The cultivated species are mostly cited with reference to their sapid qualities (binomial test, $p<0.05$ ), while aromaticity is not a feature that characterizes them significantly (binomial test, $p>0.05$ ).

\section{The cultural importance of plant landscape units}

The highest average values for cultural importance were obtained for the species found in high environments, on the stony slopes or "pedreros de la cordillera" (average consensus value * RI = 29) followed by the water bodies: "arroyos", "menucos" and "mallines" (25), while the lowest values were obtained for the units close to dwellings and other anthropized zones (20), for the dry plates: "pampas" and "lomas secas" (11), for "purchase" and "barter" (10) and for the cultivated areas (9). However, the comparative analysis of these values indicates no significant differences (Kruskal-Wallis test, $\mathrm{p}=0.65$ ). One relevant characteristic of the principal species, in terms of the indices considered, whether gathered, purchased, bartered or cultivated, is that they are aromatic and/or sapid (Table 1).

The higher cultural importance of species coming from high Andean environments coincides with reports on other Andean populations in the southern cone [33,39]. This speaks of the great therapeutic versatility of these environments, which could explain their high cultural value. Molares and Ladio [25] have highlighted the sacred character and the higher medicinal potential of the highest altitude levels for other Mapuche communities of Argentina.

\section{Medicinal species: characteristics and organoleptic classification}

Nahuelpan inhabitants mentioned 121 ethnospecies belonging to 55 botanical families, of which 108 were identified taxonomically (Table 1). The most frequently cited species were: Baccharis sagittalis (Less.) DC ("carqueja") (83.3\%), Gunnera tinctorea (Molina) Mirb. ("nalca”) (83\%), Artemisia absinthium L. ("ajenco") (72\%), Fabiana imbricata Ruiz. \& Pav. ("palo piche") (72.2\%), Adesmia boronioides Hook. f. ("paramela”) (66.7\%), Marrubium vulgare L. ("malva rubia") (55\%) and Valeriana carnosa Sm. ("ñamkulawen") (66.7\%); all these species are strongly aromatic and/or sapid.

Considering only the data on the taxonomically identified species, it was found that native species richness (59) was higher than that of exotic species (49) (binomial test, $p<0.05)$. In recent years the use of exotic species has begun to be interpreted as possibly reflecting a certain amount of flexibility on the part of individuals in order to take advantage of the changes taking place in their cultural landscape, as they incorporate new resources that contribute to their survival and wellbeing. This would indicate exploratory processes with unknown or recently discovered plants, demonstrating a certain capacity for adaptation on the part of local inhabitants [40].

Of the inhabitants interviewed, $80 \%$ mentioned that all the medicinal plants had an aroma, and that if an aromatic plant was not recognized as medicinal it was very likely that it hadn't been tested to identify its properties.

Of a total of 456 positive recordings of the presence of organoleptic characteristics of the mentioned species, it was found that $60 \%$ corresponded to flavor and $40 \%$ to aroma. There are no organoleptic data for $34 \%$ of the species registered. This could be due to the taboo associated with communicating certain symbolic aspects of traditional botanical knowledge to people from outside the community, in particular because certain strong aromas serve as vehicles or intermediaries between the natural and supernatural worlds [29].

Of the plants cited, 78 species are described by their flavor and 52 by their aroma (Table 1). In general terms informants classified the flavor as "acid"/"sour" (e.g., the whole plant of Oxalis spp.), "bitter" (e.g., the stalks of Baccharis sagittalis (Less.) DC), "spicy" (e.g., the roots of Lycium chilense Miers ex Bertero), "sweet" (e.g., the branches of Ribes cucullatum Hook. \& Arn.), and "dry"/ "like alum"/like tea" (referring to Camellia sinensis, e.g., the roots of Acaena splendens Hook. \& Arn.) (Table 1). The last mentioned character refers to the perception of astringency, which, according to Amat and Vincent [41] is a property perceived more by the sense of touch than of taste.

Aroma is perceived as "scented" (e.g. branches of Acantholippia seriphioides (A. Gray) Moldenke), "minty" (referring to Mentha spp., e.g. branches of Clinopodium darwinii (Benth.) Kuntze), "like boldo" (referring to Peumus boldus Molina, e.g. branches of Dysphania ambrosioides (L.) Mosyakin \& Clemants), "like tobacco" 
(referring to Nicotiana tabacum L., e.g. leaves of Verbascum thapsus L.), "like the smell of dirty feet" (e.g. roots and rhizomes of Valeriana carnosa Sm.), "heavy, dense" (e.g. branches of Ruta chalepensis L.), "like caramel", because of the aroma and resinous texture (e.g.: stalks of Adesmia boronioides Hook. f.).

In addition, in many cases aroma and flavor were interpreted together, and subclassified into two contrasting categories: "füre" (strong/unpleasant/disgusting, in Mapuzungun) (e.g. branches of Artemisia absinthium L.) and "mild/nice" (e.g. roots of Armeria maritima (Mill.) Willd.). In the interviews, informants were not able to distinguish between unpleasant and strong, or between mild and nice. This indistiction between the quality and the intensity of aromas and flavors may indicate the absence of linguistic labels for these perceptions [42], or possibly a fundamentally practical perceptual-functional connection.

\section{Therapeutic uses and sensorial characteristics}

In total, 526 reports of medicinal uses were registered. Of these, 25\% were related to gastro-intestinal use, 19\% to respiratory, $10 \%$ to analgesic-anti inflammatory, $9 \%$ to dermatological-cosmetic and $8 \%$ to cultural syndromes ("mal de ojo", "pasmo", "frío", "mal aire"), 6\% to febrifuges, $6 \%$ to cardio-vascular, $6 \%$ to obstetric-gynaecological, $5 \%$ to genito-urinary and 6\% to other uses (endocrine, ophtalmological, etc. $)(p<0.05)$.

It is noteworthy that certain species with mild aromas and/or flavors, recognized as "kochü lawen", are used for strongly culturally based syndromes. For example, Erodium cicutarium (L.) L'Hér. ex Aiton, a plant which has become established in the wild and is widespread in anthropized areas throughout the region, is used to treat the "pasmo", a condition that includes symptoms such as stomach pains, cramps and abnormal pigmentation of the skin, which is caused by an imbalance between the states of hot and cold; the cultivated, aromatic Melissa officinalis L. is used for "afligimiento del corazón (heart troubles)" which involves feeling sadness and a general decline in spirits; the purchased seeds of Linum usitatissimum L.are used after childbirth (for "domo kütran" or "mollfüñ kütran") which is "cuando la sangre queda coajada adentro (when coagulated blood remains inside)", a problem which presents symptoms of headaches, backaches and bleeding after the birth, and can be fatal. There are also certain problems related to the supernatural world, such as the "susto" (fright), the result of a meeting with spirits such as the "anchimallen" (who takes the form of a child), with the "cherrufes" (lights in the countryside), with the "meulen" (dangerous whirlwinds), or with the "cuero" or "wayllepen" (an animal with extended limbs which appears in isolated places close to water bodies), among others, which often require highly aromatic species in their treatment. These belong to the group of the "alwe lawen" (medicines for the souls of the dead), and include species such as Rosmarinus officinalis L., Ruta spp., Laurus nobilis L. and Artemisia absinthium L., which are considered "füre lawen" (strong, bitter, potent medicines). These species are generally used in the form of aromatic smoke, thrown on the embers of the fire; hung on the body as amulets beneath the clothing, on the walls of dwellings, or on the door thresholds as protectors or 'defensive' elements. They also tend to be grown in pots or in homegardens so as to favor the wellbeing of the family and their animals.

In order to deal with these and many other problematic situations, the informants have restructured the significance of resources which have appeared relatively recently in the region. These processes show, in part, the adaptive capacity of these indigenous communities, which comes into action when socio-cultural and ecological changes must be faced [18].

Similar to that detected in the studies carried out in communities of the sub Antarctic forest [8,25], it was found that the plants with highest use consensus, in this case used for digestive, respiratory, cardio-vascular, analgesic-anti-inflammatory, obstetric-gynaecological and genito-unrinary complaints, have the highest frequencies of cites reporting flavor (Spearman test, $p<0.05$ ), and those with the highest frequencies relating to digestive, analgesicanti-inflammatory and cultural syndromes present the highest frequencies of cites reporting aroma (Spearman test, $p<0.05$; Table 1).

\section{Conclusions}

This work on the cultural landscape of a community living in the steppe, studied from an eco-sensorial perspective, sheds new light on the ways societies perceive and manage their natural surroundings. The pattern found in this study shows that a bidirectional process is occurring; local people activities on their arid land and the subsequent environment respond are very closely connected, possibly adding, a specific organoleptic trait in each landscape unit.

Our focus on the medicinal plants available in the community enabled us to discover that the processes of cultural perception, use, selection and management of these surroundings are influenced by the organoleptic attributes of their components. These components undergo changes, that is, native and exotic wild species, species which are bartered or purchased and those cultivated in the homegardens may all enter or leave the system, depending on environmental and social circumstances that regulate the everyday lives of the inhabitants. Barter and purchase, as socially extended practices of high cultural value, are strategies and relevant sources for the supply of medicinal resources, which extends the limits of the herbal 
landscape, in such a way that other layers are added to the local medical system, which, although they do not occupy any defined area of land, expand the herbal landscape as a whole [6]. Different social-political and environmental phenomena over the last century have had an impact on the Patagonian biocultural landscape [36,43]. In this complex landscape, changeable in its components and processes, knowledge of the resources is learned in the everyday business of survival, both individually and collectively, indoors and outdoors, and is permeable to the flow of exogenous information $[18,44]$. Access to the mass media, formal education, the involvement of external social organizations, and the inclusion of more young people in the labor markets of towns in the region have favored the incorporation of knowledge and resources proceeding from the global market.

The pharmacopeia as a cultural construction of the local medical system has not been excluded from this context, but has adjusted to it through innovatory processes [45], thus providing more effective answers to health problems in its prophylactic and therapeutic role and in the diversity of psycho-physical and emotional circumstances that arise.

In this process of change, organoleptic perceptions play a significant role in the criteria of identification, selection and use of medicinal plants $[2,9,25]$, which are also fluctuating in the time and space.

According to various authors, the association between chemo-sensorial concepts and precepts and particular therapeutic functions is an underlying cognitional strategy that operates, together with other mental processes, as a heuristic tool [46-48]. It has been proposed that the heuristic part is the simple rule that guides the making of decisions and resolves practical problems. In general, a heuristic process can be considered as a short cut in complex mental processes, and is therefore a measure which economizes on mental resources while frequently arising from analogical reasoning [49]. An example of a mental short cut is the use of a stereotype or prototype, that is, an element that best expresses the characteristics of a group of elements belonging to a culturally significant domain, such as a category of medicinal use [46]. When a species is judged according to a stereotypic description of a group it is thought to belong to, the use of the stereotype can be useful. In this sense, organoleptic perceptions can be valuable features in the characterization of plant prototypes [48]. The importance of species prototypes lies in their role as cultural and personal reference points, which help individuals remember and transmit knowledge of illnesses and their treatment to other members of the population [48]. Thus, prototypes may function as brief guides in the selection of species from the flora with which each population interacts on a daily basis [11]. In this study we can show that, organoleptic perception help people in the determination of the functions of new available resources, and also in the assimilation of ideas and symbols different to their native ones. All these perceptive processes form part of the construction of cultural landscapes, and our study is crucial for a more in depth understanding of their dynamics in time and mechanisms of adaptation to deal with the socio-environmental changes faced by indigenous communities today.

\section{Competing interest}

The authors declared that they have no competing interests.

\section{Authors' contribution}

$\mathrm{SM}$ and $\mathrm{AL}$ have conceptualized the study and wrote the entire manuscript. Both authors approved the final version of the manuscript.

\section{Acknowledgements}

Our thanks is due to the inhabitants of the Nahuelpan community, for their friendly disposition and generous help. This research was supported by a post-doctoral fellowship assigned to Soledad Molares by the Consejo Nacional de Investigaciones Cientificas y Técnicas of Argentina (CONICET). It was also supported by the Fondo Nacional de Ciencia y Técnica (FONCYT, grant PICT 2012-1073) of Argentina.

\section{Author details}

${ }^{1}$ CONICET-Facultad de Ciencias Naturales, Universidad Nacional de la Patagonia San Juan Bosco, Ruta Nacional Nº 259 km 16,41, Esquel 9200 Chubut, Argentina. ${ }^{2} I N I B I O M A, C O N I C E T-$ Universidad Nacional del Comahue, Quintral 1250, Bariloche 8400, Río Negro, Argentina.

Received: 9 May 2014 Accepted: 6 August 2014

Published: 26 August 2014

\section{References}

1. Balée W: Advances in Historical Ecology. New York: Columbia University Press; 1998

2. Davidson-Hunt I, Berkes F: Learning as you journey: Anishinaabe perception of social-ecological environments and adaptive learning. Conserv Ecol 2003, 8:5.

3. Berkes F, Turner N: Knowledge, learning and the resilience of socialecological systems. Hum Ecol 2006, 34:479-494.

4. Toledo V, Bassols N: La memoria Biocultural. La Importancia Ecológica de las Sabidurías Tradicionales. Barcelona: Ed. Icaria; 2010.

5. Capparelli A, Hilgert N, Ladio A, Lema VS, Llano C, Molares S, Pochettino ML, Stampella P: Paisajes culturales de Argentina: pasado y presente desde las perspectivas etnobotánica y paleoetnobotánica. Rev Asoc Argent Ecol Paisajes 2011, 2:67-79.

6. Sõukand R, Kalle R: Herbal landscape: the perception of the landscape as a source of medicinal plants. Trames 2010, 14:207-226.

7. Lema VS: Criar y ser Criados por las Plantas y sus Espacios en los Andes Septentrionales de Argentina. In Espacialidades Altoandinas. Avances de Investigación Desde el Noroeste Argentino. Edited by Benedetti A, Tomasi J. Buenos Aires: Eudeba; 2013:1-20.

8. Molares S, Ladio A: Mapuche perceptions of Andean Nothofagus forests and their medicinal plants: a case study from a rural community in Patagonia, Argentina. Biodivers Conserv 2012, 21:1079-1093.

9. Shepard $\mathrm{GH}$ : A Sensory ecology of medicinal plant therapy in two amazonian societies. Am Anthropol 2004, 106:252-266.

10. Molares S, González S, Ladio AH, Castro MA: Etnobotánica, anatomía y caracterización físico-química del aceite esencial de Baccharis obovata Hook. et Arn. (Asteraceae: Astereae). Acta Bot Bras 2009, 23:578-589.

11. Molares S: Flora Medicinal Aromática de la Patagonia: Características Anatómicas y Propiedades Organolépticas Utilizadas en el Reconocimiento por Parte de la Terapéutica Popular, PhD Thesis. S.C. de Bariloche: Universidad Nacional del Comahue; 2010

12. Dillehay TD, Navarro HX: Explotación y uso de los recursos marinos y patrones de residencia entre los mapuches: algunas implicaciones preliminares para la arqueología. Maguaré 2003, 17:260-273. 
13. Johns T: With Bitter Herbs they shall Eat it: Chemical Ecology and the Origins of Human Diet and Medicine. Tucson AZ: University of Arizona Press; 1990.

14. Hutchinson B, Suárez E, Fortunato R, Beeskow AM, Bye R, Montenegro G, Timmermann B: Conservation and ethnobotanical programs of the bioactive agents from dryland biodiversity of Latin American project. Arid Land Newsletter 2000, 48:1-7.

15. Almeida CFC, Silva TCL, Amorin ELC, Maia MBS, Albuquerque UP: Life strategy and chemical composition as predictors of the selection of medicinal plants from the Caatinga (Northeast Brazil). J Arid Environ 2005, 62:127-142

16. Molares S, Ladio AH: Criterios Ambientales y Organolépticos en los Patrones de Selección y uso de Plantas Medicinales en una Comunidad Mapuche de la Patagonia Semiárida Argentina. In Traditions and Transformations in Ethnobotany. Edited by Pochettino ML, Ladio AH, Arenas P. La Plata: CYTED; 2010:286-290.

17. Ladio A, Lozada M: Patterns of use and knowledge of wild edible plants from distinct ecological environments: a case study of a Mapuche community from NW Patagonia. Biodivers Conserv 2004, 13:1153-1173.

18. Richeri M, Ladio AH, Beeskow AM: Conocimiento tradicional y autosuficiencia: la herbolaria rural en la Meseta Central del Chubut (Argentina). BLACPMA 2013, 12:44-58

19. Ladio A, Lozada $M$, Weigandt $M$ : Comparison of traditional wild plants use between two Mapuche communities inhabiting arid and forest environments in Patagonia, Argentina. J Arid Environ 2007, 69:695-715.

20. Eyssartier C, Ladio AH, Lozada M: Traditional horticultural and gathering practices in two semi-rural populations of Northwestern Patagonia. J Arid Environ 2013, 97:18-25.

21. Stepp JR: The role of weeds as sources of pharmaceuticals. J Ethnopharmacol 2004, 92:163-166

22. Correa MN: Flora Patagónica, Parte I. Buenos Aires: Colección Científica del INTA; 1998.

23. Díaz Fernández A: Descripción del Mapuzungun Hablado en las Comunidades del Departamento Futaleufú: Lago Rosario-Sierra Colorada y Nahuelpán, PhD Thesis. Bahía Blanca: Universidad Nacional del Sur; 2004

24. Scarpa GF: Plantas empleadas contra trastornos digestivos en la medicina tradicional criolla del Chaco noroccidental. Dominguezia 2002, 18:36-50.

25. Molares S, Ladio A: Ethnobotanical review of the Mapuche medicinal flora: Use patterns on a regional scale. J Ethnopharmacol 2009, 122:251-260

26. Cunningham AB: Ethics, Ethnobiological Research and Biodiversity, Research Report. Switzerland: WWF-International; 1993.

27. Silva TC, Cruz P, Araújo AS, Schwarz ML, Albuquerque UP: Methods in Research of Environmental Perception. In Methods and Techniques in Ethnobiology and Ethnoecology. Edited by Albuquerque UP, Lucena RFP, Cunha LV FC, Alves RRN. New York: Humana Press; 2014:99-109.

28. Zuloaga FO, Morrone O, Belgrano MJ: Catálogo de las plantas vasculares del Cono Sur (Argentina, Sur de Brasil, Chile, Paraguay y Uruguay), Monographs in Systematic Botany from the Missouri Botanical Garden, Volume 107. St. Louis: Missouri Botanical Garden Press; 2008.

29. Molares S, Ladio A: Chemosensory perception and medicinal plants for digestive ailments in a Mapuche community in NW Patagonia, Argentina. J Ethnopharmacol 2009, 123:397-406.

30. Bennett BC, Prance GT: Introduced plants in the indigenous pharmacopeia of Northern South America. Econ Bot 2000, 54:90-102.

31. Bran D, Gaitán J, Ayesa J, López C: La Vegetación de los Mallines del Noroeste de Patagonia. Bariloche: Informe Técnico del INTA; 2004

32. Santos JP, Araujo EL, Albuquerque UP: Richness and distribution of useful woody plants in the semi-arid region of northeastern Brazil. J Arid Environ 2008, 72:652-663.

33. Thomas E, Vandebroek I, Van Damme P, Goetghebeur P, Douterlungne D, Sanca S, Arrazola S: The relation between accessibility, diversity and indigenous valuation of vegetation in the Bolivian Andes. J Arid Environ 2009, 73:854-861.

34. Toursarkissian M: Plantas Medicinales de la Argentina. Sus Nombres Botánicos, Vulgares, Usos y Distribución Geográfica. Buenos Aires: Hemisferio Sur; 1980.

35. Reyes-García V, Vadez V, Huanca T, Leonard W, Wilkie D: Knowledge and consumption of wild plants: a comparative study in two Tsimane' villages in the Bolivian Amazon. Ethnobot. Res App/ 2005, 3:201-207.

36. Reis MS, Ladio AH, Peroni N: Landscapes with Araucarias in South America: Evidence for a Cultural Dimension. Ecol Soc 2014, in press.
37. Cardoso MB, Ladio AH: Forestación peridoméstica en Patagonia y conocimiento ecológico tradicional: un estudio de caso. Sitientibus Sér Ciênc Biol 2011, 11:321-327.

38. Rovere $A E$, Molares S, Ladio AH: Plantas utilizadas en cercos vivos de ciudades patagónicas: aportes de la etnobotánica para la conservación. Ecol Austral 2013, 23:165-173.

39. Martinez MR, Pochettino ML: Microambientes y Recursos vegetales terapéuticos. Conocimiento local en Molinos, Salta, Argentina. Zonas Áridas 2004, 8:18-31.

40. Ladio AH: Traditional knowledge of edible wild native and exotic plants in the context of cultural change in human populations of arid Patagonia. Biorem Biodiv Bioavail 2011, 5:60-64.

41. Amat JM, Vincent JD: Una Nueva Fisiología del Gusto. Barcelona: RBA Libros; 2003.

42. Pieroni A, Nebel S, Quave C, Münz H, Heinrich M: Ethnopharmacology of liakra: traditional weedy vegetables of the Arbëreshë of the Vulture area in southern Italy. J Ethnopharmacol 2002, 81:165-185.

43. Torrejón F, Cisternas M, Araneda A: Efectos ambientales de la colonización española desde el río Maullín al archipiélago de Chiloé, sur de Chile. Rev Chil Hist Nat 2004, 77:661-677.

44. Molares S, Ladio A: The usefulness of edible and medicinal Fabaceae in Argentine and Chilean Patagonia: environmental availability and other sources of supply. eCAM 2012, doi: 10.1155/2012/901918.

45. Pochettino ML, Lema VS: La variable tiempo en la caracterización de conocimiento botánico tradicional. Darwiniana 2008, 46:227-239.

46. Rosch E: Principles of Categorization. In Cognition and Categorization. Edited by Rosch E, Lloyd B. Hillsdale: Lawrence Erlbaum Assoc.; 1978.

47. Berlin B: Ethnobiological Classification: Principles of Categorization of Plants and Animals in Traditional Societies. Princeton University Press: Princeton; 1992.

48. Casagrande DG: Human taste and cognition in Tzeltal Maya medicinal plants use. J Ecol Anthropol 2002, 4:57-69.

49. Moustakas C: Heuristic Research: Design, Methodology, and Applications. Newbury Park, CA: Sage Publications, Inc:i 1990

doi:10.1186/1746-4269-10-61

Cite this article as: Molares and Ladio: Medicinal plants in the cultural landscape of a Mapuche-Tehuelche community in arid Argentine Patagonia: an eco-sensorial approach. Journal of Ethnobiology and Ethnomedicine 2014 10:61

\section{Submit your next manuscript to BioMed Central and take full advantage of:}

- Convenient online submission

- Thorough peer review

- No space constraints or color figure charges

- Immediate publication on acceptance

- Inclusion in PubMed, CAS, Scopus and Google Scholar

- Research which is freely available for redistribution 